\title{
Some Newly Defined Sequence Spaces Using Regular Matrix of Fibonacci Numbers
}

\section{ShyamalDebnath, SubrataSaha}

Tripura University, Department of Mathematics, Agartala, India. e-mail:shyamalnitamath@gmail.com

Arrival date:31.10.2013; Accepted date:06.01.2014

\author{
Key words \\ Fibonacci Number; \\ Regular Matrix; \\ Sequence Space. \\ 11B39, 46B45
}

\begin{abstract}
The main purpose of this paper is to introduce the new sequence spaces $c_{0}(F), c(F)$ and $l_{\alpha}(F)$ based on the newly defined regular matrix $F$ of Fibonacci numbers. We study some basic topological and algebraic properties of these spaces. Also we investigate the relations related to these spaces.
\end{abstract}

\section{Introduction}

Let $w$ be the space of all real sequences. Any vector subspace of $w$ is called a sequence space. We shall write $c, c_{0}$ and $l_{\infty}$ for the sequence spaces of all convergent, null and bounded sequences.

Let $X, Y$ be two sequence spaces and $A=\left(a_{n k}\right)$ be an infinite matrix of real numbers $a_{n k}$, where $n, k \in$ $N$.Then, A defines a matrix mapping (Debnath and Debnath, communicated; Malkowsky and Rakocevic, 2007) from $X$ into $Y$ and we denote it by $A: X \rightarrow Y$, if for every sequence $X=\left(x_{k}\right) \in X$, the sequence $A x=\left\{A_{n}(x)\right\}_{n=1}^{\infty}$, the $A$-transform of $x$, is in $Y$; where

$$
A_{n}(x)=\sum_{k=1}^{\infty} a_{n k} x_{k},(n \in N)
$$

By $(X, Y)$, we denote the class of all matrices $A$ such that $A: X \rightarrow Y$. Thus $A \in(X, Y)$ if and only if the series on the right hand side above converges for each $n \in N$ and every $x \in X$ and we have $A x \in Y$ for all $x \in X$. The matrix domain $X(A)$ of an infinite matrix $A$ in a sequence space $X$ is defined by

$X(A)=\left\{x=\left(x_{k}\right) \in W: A x \in X\right\}$,

which is a sequence space (Altay, Basar and Mursaleen, 2006; Kara and Basarir, 2012; Mursaleen and Noman, 2010; Tripathy and Sen, 2002).

A sequence space $X$ is called $F K$ space if it is a complete linear metric space with continuous (c) Afyon Kocatepe Üniversitesi

coordinates $p_{n}: X \rightarrow R(n \in N)$, where $R$ denotes the real field and $p_{n}(x)=x_{n}$ for all $x=\left(x_{k}\right) \in X$ and every $n \in N$. A $B K$ space is a normed $F K$ space, i.e, a $B K$ space is a Banach space with continuous coordinates. The spaces $c, c_{0}$ and $l_{\infty}$ are $B K$ spaces with $\|x\|=\sup _{k}\left|x_{k}\right|$.

The following lemma ( Known as The Toeplitz Theorem) contains necessary and sufficient condition for regularity of a matrix.

Lemma 1.1(Wilansky, 1984): Matrix $A=\left(a_{n k}\right)_{n, k=1}^{\infty}$ is regular if and only if the following three conditions hold:

(1) There exists $M>0$ such that for every $n=1,2, \ldots$ the following inequality holds:

$$
\sum_{k=1}^{\infty}\left|a_{n k}\right| \leq M
$$

(2) $\lim _{n \rightarrow \infty} a_{n k}=0$ for every $k=1,2, \ldots$

(3) $\lim _{n \rightarrow \infty} \sum_{k=1}^{\infty} a_{n k}=1$.

Let $\left(p_{k}\right)$ be a sequence of positive numbers and $P_{n}$ $=\sum_{k=1}^{n} p_{k}$.

Then the matrix $R^{p}=\left(r_{n k}^{p}\right)$ of the Riesz mean is given by

$r_{n k}^{p}=\left\{\begin{array}{c}\frac{p_{k}}{P_{n}}, \text { if } 1 \leq k \leq n ; \\ 0, \text { otherwise }\end{array}\right.$

It is known that the Riesz matrix is a Toeplitz matrix if and only if $P_{n} \rightarrow \infty$ as $n \rightarrow \infty$ (Basar, 2011). 
The Fibonacci numbers (Kara and Basarir, 2012; Koshy, 2001) are the sequence of numbers

$\left\{f_{n}\right\}_{n=1}^{\infty}$ defined by the linear recurrence equations

$f_{0}=0$ and $f_{1}=1, f_{n}=f_{n-1}+f_{n-2} ; n \geq 2$.

Fibonacci numbers have many interesting properties and applications in arts, sciences and architecture. Also, some basic properties of Fibonacci numbers are given as follows (Kalman and Mena, 2003; Vajda, 1989):

$\sum_{k=1}^{n} f_{n}=f_{n+2}-1 ; n \geq 1$,

$\sum_{1}^{n} f_{n}^{2}=f_{n} f_{n+1} ; n \geq 1$,

$\sum_{k=1}^{\infty} \frac{1}{f_{k}}$ converges.

In this paper, we define the Fibonacci matrix $F=$ $\left(f_{n k}\right)_{n, k=1}^{\infty}$, which differs from existing Fibonacci matrix by using Fibonacci numbers $f_{n}$ (Kara and Basarir, 2012) and introduce some new sequence spaces related to matrix domain of $F$ in the sequence spaces $c_{0}, c$ and $l_{\infty}$.

\section{Main Result}

Now, we define the Fibonacci matrix $F=\left(f_{n k}\right)_{n, k=1}^{\infty}$, by

$$
f_{n, k}=\left\{\begin{array}{c}
\frac{f_{k}}{f_{n+2}-1}(1 \leq k \leq n) \\
0, \text { ot lerwise }
\end{array}\right.
$$

that is,

$$
\left[\begin{array}{cccccc}
1 & 0 & 0 & 0 & 0 & \ldots \\
\frac{1}{2} & \frac{1}{2} & 0 & 0 & 0 & \ldots \\
\frac{1}{4} & \frac{1}{4} & \frac{2}{4} & 0 & 0 & \ldots \\
\frac{1}{7} & \frac{1}{7} & \frac{2}{7} & \frac{3}{7} & 0 & \ldots \\
\vdots & \vdots & \vdots & \vdots & \vdots & \ddots
\end{array}\right]
$$

It is obvious that the matrix $F$ is triangular matrix i.e, $f_{n n} \neq 0$ for $k \leq n$ and $f_{n k}=0$ for $k>n$ $(n=1,2,3, \ldots)$. Also it follows from the lemma 1.1 that the method $\mathrm{F}$ is regular.
Now, we introduce the following sequence spaces based on the infinite matrix $F$ :

$c(F)=\left\{x=\left(x_{k}\right) \in w: F x \in c\right\}$

$c_{0}(F)=\left\{x=\left(x_{k}\right) \in W: F x \in c_{0}\right\}$

$l_{\infty}(F)=\left\{x=\left(x_{k}\right) \in W: F x \in l_{\infty}\right\}$

where $F x=\left\{F_{n}(x)\right\}_{n=1}^{\infty}$ and $F_{n}(x)=\sum_{k=1}^{\infty} f_{n k} x_{k}$ $=\frac{1}{f_{n+2}-1} \sum_{k=1}^{n} f_{n k} x_{k},(n \in N)$.

Theorem 2.1: The spaces $c(F), c_{0}(F)$ and $l_{\infty}(F)$ are $B K$ spaces with the same norm given by

$\|x\|_{X(F)}=\|F x\|_{X}=\sup _{n}\left|F_{n}(x)\right|$

where $X \in\left\{c, c_{0}, l_{\infty}\right\}$.

Proof: By Theorem 4.3.12 of Wilanksy, 1984 [p.63] and as the matrix $F$ is triangular, we have the result.

Remark 2.2: It can be easily seen that the absolute property does not hold on the spaces $c(F), c_{0}(F), l_{\infty}(F)$ i.e., $\|x\|_{X(F)} \neq\||x|\|_{X(F)}$ for at least one sequence $\mathrm{x}$ in each of these spaces, where $|x|=\left(\left|x_{k}\right|\right)$. Thus the spaces $c(F), c_{0}(F)$ and $l_{\infty}(F)$ are $B K$ spaces of non-absolute type.

Theorem 2.3: The sequence spaces $c(F), c_{0}(F)$ and $l_{\infty}(F)$ are norm isomorphic to the spaces $c, c_{0}$ and $l_{\infty}$, respectively i.e, $c(F) \cong c, c_{0}(F) \cong c_{0}$ and $l_{\infty}(F)$ $\simeq l_{\infty}$.

Proof: $X$ denotes any of the spaces $c, c_{0}$ or $l_{\infty}$ and $X(F)$ be the respective one of the spaces $c(F), c_{0}(F)$ or $l_{\infty}(F)$. Since the matrix $F$ is triangular, it has a unique inverse, which is also triangular (Wilansky, 1984, proposition 1.1). Therefore the linear operator $L_{F}: \mathrm{X}(\mathrm{F}) \rightarrow \mathrm{X}$, defined by $L_{F}(x)=F(x)$ for all $x \in X(F)$, is bijective and is norm preserving by above norm in theorem 2.1. Hence $X(F) \cong X$.

Theorem 2.4: The inclusions $c_{0}(F) \subset c(F) \subset l_{\infty}(F)$ strictly hold.

Proof: It is clear that the inclusion $c_{0}(F) \subset \mathrm{c}(F)$ $\subset l_{\infty}(F)$ hold. 
Consider the sequence $x=\left(x_{k}\right)$ defined by $x_{k}=1$, for all $k \in N$. Then we have for every $n \in N$,

$F_{n}(x)=\frac{1}{f_{n+2}-1} \sum_{k=1}^{n} f_{k}=1$

This shows that $F x \in c$ but not in $c_{0}$. Thus the sequence $x$ is in $c(F)$ but not in $c_{0}(F)$. Hence the inclusion $c_{0}(F) \subset \mathrm{c}(F)$ strictly holds.

Again, consider the sequence $x=\left(x_{k}\right)$ defined by $x_{k}=\frac{(-1)^{k}\left(f_{k+2}+f_{k+1}-1\right)}{f_{k}}$, for all $k \in N$.

Then we have for every $n \in N$,

$F_{n}(x)=\frac{1}{f_{n+2}-1} \sum_{k=1}^{n} f_{k} x_{k}=(-1)^{n}$

This shows that $F x \in l_{\infty}$ but not in $c$. Thus the sequence $x$ is in $l_{\infty}(F)$ but not in $c(F)$. Hence the inclusion $c(F) \subset l_{\infty}(F)$ strictly holds.

Theorem 2.5: The inclusion $c_{0} \subset c_{0}(F), c \subset c(F)$ and $l_{\infty} \subset l_{\infty}(F)$ holds.

Proof: As $F$ is a regular matrix, so the inclusion $c_{0} \subset c_{0}(F)$ and $c \subset c(F)$ are obvious.

Now, let $x=\left(x_{k}\right) \in l_{\infty}$. Then there is a constant $M$ $>0$ such that $\left|x_{k}\right| \leq M$ for all $k \in N$. Thus for each $n$ $\in N$

$\left|F_{n}(x)\right| \leq \frac{1}{f_{n+2}-1} \sum_{k=1}^{n} f_{k}\left|x_{k}\right|$

$\leq \frac{M}{f_{n+2}-1} \sum_{k=1}^{n} f_{k}=M$

which shows that $F x \in l_{\infty}$ i.e., $x \in l_{\infty}(F)$. Thus we conclude that $l_{\infty} \subset l_{\infty}(F)$.

Example: Consider the sequence $\mathrm{x}=\left(x_{k}\right)=(1,0,1$, $0,1,0$, .). Then we have for every

$n \in N$

$F_{n}(x)=\frac{1}{f_{n+2}-1} \sum_{k=1}^{n} f_{k} x_{k}=\frac{1}{f_{n+2}-1}\left(f_{1}+f_{3}+\ldots\right.$ $\left.+f_{n}\right)$

which is convergent.

This shows that $F x \in C$ but $x$ is not in $c$. Thus the sequence $x$ is in $c(F)$. Hence the inclusion $c \subset c(F)$ strictly holds.

Similarly, we can show the other inclusions are strict.

\section{References}

Altay B., Basar F. and Mursaleen M., 2006. On the Euler sequence spaces which include the spaces $l_{p}$ and $l_{\infty}$ I, Informations Science, 176, 1450-1462.

Basar F., 2011. Summability Theory and Its Applications, Bentham Science Publishers, Istanbul.

Debnath S. and Debnath J., On I-statistically convergent sequence spaces defined by sequences of Orlicz functions using matrix transformation (Communicated).

Kara E. E. and Basarir M., 2012. An application of Fibonacci numbers into infinite Toeplitz matrices, CJMS. 1(1), 43-47.

Kalman D. and Mena R., June 2003. The Fibonacci numbers-Exposed, Mathematics Magazine. 76(3).

Koshy T., 2001. Fibonacci and Lucas Numbers with Applications, Wiley.

Mursaleen M. and Noman A. K., 2010. On the space of $\lambda$-convergent and bounded sequences, Thai J. Math. 8(2), 311-329.

Malkowsky E. and Rakocevic V., 2007. On matrix domains of triangles, Appl. Math.Comput., 189(2), 1146-1163

Tripathy B. C. and Sen M., 2002. On a new class of sequences related to the space $l_{p}$, Tamkang J. Math. 33(2), 167-171.

Vajda S., 1989. Fibonacci and Lucas Numbers, and Golden Section: Theory and Applications, Chichester: Ellis Horword.

Wilansky A., 1984. Summability through functional analysis, North-Holland mathematics Studies 85, Elsevier Science Publishers, Amsterdam: New York: Oxford. 